\title{
Valentin Ickelsamers Beitrag zum Deutschunterricht
}

1. Ziele und Aufgaben des neuen Unterrichts

2. Die Grundlegung des neuen Unterrichts

3. Der dem neuen Unterricht implizite Lehrplan

3.1. Die lautanalytischen Übungen

3.2. Die Übungen im Schreiben

3.3. Übungen im Lesen

3.4. Orthographische Übungen

3.5. Übungen in der deutschen Grammatik

3.6. Übungen in der Bestimmung der Herkunft und Zusammensetzung deutscher Wörter (Etymologie und Wortbildung)

4. Ergebnisse der Untersuchung

Von Valentin Ickelsamer (etwa 1500 bis 1547), einem süddeutschen Schulmeister, über dessen Leben wir nur unzureichend unterrichtet sind (Müller 1882; Vogel 1894; Giesecke 1992), stammen zwei kleinere Schriften, die seit geraumer Zeit die Aufmerksamkeit auf sich gezogen haben: „Die rechte weis aufs kürtzist lesen zu lernen“ (1527) und „Ain Teütsch Grammatica“ (um 1534). Von beiden gibt es mehrere Ausgaben und Auflagen.

Die Ausführungen in diesen Schriften stehen in drei unterschiedlichen Zusammenhängen. Zunächst beanspruchen sie nicht mehr zu sein als eine Anweisung zum Lesenlernen. Das erste Büchlein widmet sich ausschließlich dieser Aufgabe, das zweite greift zumindest auch diese Frage auf und merkt dies ausdrücklich im Untertitel an: „Ain Teütsche Grammatica, darauß ainer von jm selbs mag lesen lernen“. So sind beide also zunächst einmal in die Tradition der Didaktik und Methodik des Lesens einzuordnen. Das zweite Büchlein geht aber über diesen Rahmen weit hinaus. Es beansprucht „ain teütsche Grammatica“ zu sein und ist damit in die Tradition der Grammatikschreibung zu stellen, wie sie aus der Antike überkommen war. Donatus und Priscianus hatten die Modelle geliefert, die sie prägen sollten. - Es kommt aber noch ein weiterer Zusammenhang hinzu - keine Tradition, auf die Ickelsamer hätte zurückgreifen können, da er sie selbst begründet hat: der Unterricht in der deutschen Sprache. Von Ickelsamer führt eine ziemlich kontinuierliche Traditionslinie $\mathrm{zu}$ dem, was wir heute als Deutschunterricht bezeichnen.

Die drei Traditionen haben in der Forschung unterschiedliche Beachtung erfahren (Giesecke 1992, 169 f.). Ickelsamer stellt sich selbst ausdrücklich und mit Nachdruck in die erste Traditionslinie: 
Da ich erkandte das mich Gott über dises sein ampt setzen wolt/ das lesewerck zůgebrauchen in seinem hof und regiment auff diser erden/ hab ich nach dem vrsprung des lesens gedacht/

das hatt mir Gott so klar zaiget/

das ich nit achten kan/

das dise kunst hôher gefürt werden/

oder jrem vrsprung nẳher kommen künd/

zů welchem alle ding (wie man sagt) wider kummen sollen und můssen

(Müller 1882: 123).

So ist es denn auch verständlich, daß diese Tradition in der Forschung als erste die Aufmerksamkeit auf sich zog und inzwischen auch am gründlichsten untersucht worden ist (Göckelbecker 1931; Giesecke 1979; 1992). - Das Interesse der Sprachwissenschaftler galt von Anfang an mehr den Ausführungen zu einer Grammatik der deutschen Sprache (Socin 1888; Jellinek 1913; Noll 1935). Zu einer genaueren Untersuchung dieser Traditionslinie ist es aber erst in jüngster Zeit gekommen (Rössing-Hager 1983). - Was noch aussteht, ist eine Untersuchung der Tradition, die Ickelsamer allererst begründete: sein Beitrag zum Deutschunterricht (bemerkenswert in dieser Richtung allerdings eine in Vergessenheit geratene Dissertation: Vogel 1894). Seine Idee von einem solchen, wie er aufzubauen sei, welche Inhalte er habe und vor allem welches Ziel, wird Gegenstand der folgenden Untersuchung sein. Es geht um ein noch nicht aufgearbeitetes Kapitel der Geschichte des Deutschunterrichts.

\section{Ziele und Aufgabe des neuen Unterrichts}

Eigentlich hatte Ickelsamer nicht mehr beabsichtigt, als den Aufgabenbereich eines Elementarschullehrers zu erweitern:

Dann es ist ser vnrecht/ das die teütschen Schůlmaister nit mer künden oder thůn wôllen/ dann ainen jungen lesen/ schreiben/ vnd rechnen leren/ vnd jn dar nach nit hơher im teütschen künden füren oder leren (Müller 1882, 151).

In Wirklichkeit hat er aber ein neues Unterrichtsfach geschaffen:

Dann was ists anders/

das sich ainer auß thůt ain teütscher schůlmayster zusein/ dann ainen lerer der teütschen sprach zů sein? da nit allain lesen/schreiben/vnnd rechen zůgehơrt/ sonder ain künstlicher verstand der gantzen teütschen wo̊rter sprach art vnnd weiß? (ebd.).

Aus dem Elementarschullehrer sollte ein „Lehrer der deutschen Sprache“, aus dem Elementarunterricht, der sich auf Lesen, Schreiben und Rechnen beschränkte, ein Unterricht in der deutschen Sprache werden. Beide sollten die Schüler (und wohl auch Schülerinnen) „höher im Deutschen führen“.

Den Worten Ickelsamers zu entnehmen, der Elementarschulunterricht wäre nicht „höher“ geführt worden, wäre unzutreffend. Er wurde weiterge- 
führt, und zwar im Lateinunterricht an den Pfarr-, Stadt- und Lateinschulen. Dort wurden die klassischen Schriftsteller gelesen: in ihrem originalen Wortlaut, und es wurde auch fleißig geschrieben, wenn auch nur in lateinischer Sprache: Reden vor allem, aber auch Briefe und Gedichte. Außerhalb der Schule gab es die Möglichkeit, sich bei einem professionellen Schreiber in der Abfassung von Briefen und Urkunden unterrichten zu lassen (Kintzinger 1990, 470-472 und 373-381). Wenn Ickelsamer davon spricht, daß ein deutscher Schulmeister seine Schüler „höher führen“ solle, dann meint er weder die Weiterführung des Elementarunterrichts im Lateinunterricht der höheren Schulen noch einen ergänzenden Unterricht in der Kunst, Urkunden und Briefe aufsetzen zu können. Er bleibt im Rahmen des Elementarunterrichts, schlägt aber eine andere Richtung ein, in der die Schüler „höher“ zu führen seien. Welches Ziel er dem Unterricht an Elementarschulen gesetzt hat, erklärt er an einer Stelle, die sich nur in der Augsburger Ausgabe findet:

es bedürffen und gebrauchen die teutschen kainer andern Grammatic/ dann die kunst und vndterweysung/

recht und gůt teütsch zů reden/ vnd schreiben (Pohl 1971, A2r).

Ickelsamer denkt an einen Unterricht ausschließlich in der deutschen Sprache. Eine Grammatik, die einem solchen Unterricht zugrunde zu legen wäre, hätte dann die Aufgabe zu erfüllen, das Wissen („kunst“) zur Verfügung zu stellen und sowohl didaktisch als auch methodisch zu vermitteln (,undterweysung"), wie man richtig und gut Deutsch spricht und schreibt, also in etwa das, was wir heute von einer guten deutschen Grammatik auch erwarten würden: eine fundierte und pädagogisch durchdachte Beschreibung des Deutschen.

„Recht und gut" ist die Übersetzung von lateinisch „recte et bene“, eine Formel, die häufig in diesem Zusammenhang gebraucht wird. „Recht“ (recte) meint den korrekten, „gut“ (bene) den angemessenen Gebrauch der deutschen Sprache. Der richtige Gebrauch ist Gegenstand einer Grammatik, der angemessene Gegenstand einer Rhetorik oder Stilistik. Grammatik und Rhetorik (Stilistik) sind bei Ickelsamer, wie Rössing-Hager (1983) auch an anderen Stellen feststellen konnte, noch nicht geschieden. Seine Grammatik ist im Grunde eine rhetorische Grammatik und als rhetorische ist sie auch eine pädagogische. Aus den Aufgaben der Grammatik ergibt sich ohne weiteres ein Ziel des Grammatikunterrichtes. Seine Aufgabe ist es, die Schüler so weit zu führen, da $\beta$ sie das Deutsche in Wort und Schrift beherrschen, und sie beherrschen es dann, wenn sie sich grammatisch richtig (,recht“) und stilistisch angemessen (,gut“) auszudrücken verstehen. Das ist etwas anderes, als was sie im Lateinunterricht oder bei einem Privatlehrer lernen konnten.

$\mathrm{Zu}$ dem Wissen, wie man etwas tut (knowing how), kommt nach den Vorstellungen Ickelsamers aber noch das Wissen, was man denn da tut (knowing that). Die Kinder sollten nicht nur lernen, wie man die deutsche Sprache richtig und gut gebraucht, sie sollten auch lernen, ,jre sprach bas verstehn“ (Müller 1882, 149). Zu dem sprachlichen Vermögen, das bei den Kindern ausgebildet werden soll, tritt also als weiteres Lernziel ein besseres Verständnis 
für die deutsche Sprache, in etwa das, was die Sprachdidaktiker heute als Sprachreflexion bezeichnen würden. Die Notwendigkeit eines solchen Lernzieles wird dreifach begründet - pragmatisch, emotional und religiös-moralisch:

Mócht einer sagen/

warzů solliches zůwissen nutz wer?

Antwurt/

Wenn man solliches nit brauchet/

wie gesagt/

so kummen die worrter vnd sprach leicht in ain vergeß vnd vnverstand/

wie geschehen.

Zum andern/

so gibt soliches ain grosse lieb/

vnnd gleich ain verwunderung/

wie alle ding so fein unnd kunstlich also mit namen geziert vnd angezaigt sein/

Es dienet auch ainem verstendigen gottfürchtigen menschen zur besserung

(Müller 1882, 150).

Die Begründung greift weit aus. Die Pflege der eigenen Sprache, die Liebe zu ihr und ihre Bedeutung für das Leben eines Christen werden bemüht, um den Anspruch zu rechtfertigen, da $ß$ es in der Schule nicht nur darum geht, daß die Kinder richtig und gut zu reden und zu schreiben lernen, sondern daß dazu in gleicher Weise auch die Kenntnis und das Verständnis der deutschen Sprache gehören. Wären Ickelsamers Vorstellungen verwirklicht worden, hätten sie in der damaligen Unterrichtspraxis zu einigen einschneidenden Veränderungen geführt.

Sie hätten (1) den Rahmen des Elementarunterrichtes erheblich erweitert. Aus dem Elementarunterricht, der sich auf die elementaren Fähigkeiten Lesen, Rechnen und Schreiben beschränkte, wäre eine Art sprachlicher Grundbildung geworden, die sich durchaus mit dem hätte vergleichen können, was an den weiterführenden Schulen angeboten wurde. Es wäre also zu fragen, ob es dann überhaupt noch sinnvoll ist, von einem „Elementarunterricht“ zu sprechen.

(2) Das Lehrerbild wäre zu revidieren gewesen. Aus dem Schreib- und Rechenlehrer wäre „,ain lerer der teütschen sprach“: ,ain teütscher Schulmaister" geworden. Dessen Aufgabe ist es nicht so sehr, den Kindern Lesen und Schreiben beizubringen, als vielmehr sie mit der ,gantzen teütschen wörter sprach art und weiß" vertraut zu machen. In dem deutschen Schulmeister hätte man durchaus schon die Züge des Deutschlehrers erkennen können.

Die bedeutsamste und für Ickelsamer wichtigste Veränderung aber hätte (3) die Sprache in der Schule betroffen. Deutsch wäre nicht nur die Sprache gewesen, in der der Unterricht vorgenommen wird, Deutsch wäre vielmehr auch zum Gegenstand von Unterricht geworden, den Fremdsprachen, die an den Pfarr-, Stadt- und Lateinschulen gelehrt wurden, dem Rang nach durchaus an die Seite zu stellen.

Eine letzte Veränderung, die sich (4) abzeichnet, aber leicht übersehen wird, ergibt sich aus den vorher angeführten Punkten: das Mündliche tritt aus seinem Schattendasein heraus. Bisher war der Elementarunterricht auf Lesen 
und Schreiben, also auf schriftliche Aspekte der Sprache, beschränkt. Wenn man es aber ernst mit der Forderung meinte, daß „die ganze deutsche Sprache" Gegenstand des Unterrichtes werden solle, dann gab es keinen Grund, das Mündliche hinter dem Schriftlichen zurücktreten zu lassen.

\section{Dịe Grundlegung des neuen Unterrichts}

Den neuen Unterricht auf eine solide Grundlage stellen hieß für Ickelsamer, die der deutschen Sprache eigenen und für sie relevanten Laute zu erfassen, $\mathrm{zu}$ beschreiben und zu klassifizieren, d. h. die Erfassung der kleinsten bedeutungstragenden Einheiten, ihre Beschreibung durch die Angabe sowohl der Artikulationstelle im Mundraum als auch der Artikulationsart und ihre Klassifizierung nach Kriterien, die aus der Qualität der Laute gewonnen wird (ausführlicher dazu Vogel 1894, 36-48). Für sich genommen waren diese Operationen nicht unbekannt. Die Laute waren in dem lateinischen Alphabet schriftlich erfaßt (Genaueres dazu unten). Beschreibungen der einzelnen Laute nach ihren artikulatorischen Eigenschaften hatte es verschiedentlich schon vor Ickelsamer gegeben (Müller 1882, 406), so bei Terentianus Maurus (3. Jh. n. Chr.), Martinus Capella (5. Jh.), und Aventin (1517). Eine mögliche Klassifizierung der Laute konnte man bei Priscian in seiner großen Grammatik „De institutione grammatica" (Buch I,2) finden (Kiepe 1983, 456).

Ickelsamer hat ohne Zweifel aus dem reichen Fundus der gelehrten Tradition geschöpft, und so werden ihm einige der angeführten Quellen bekannt gewesen sein. Von Interesse ist hier, was er aus ihnen gemacht hat. Generell kann man feststellen, daß er das disparate Wissen zusammengeführt, aufeinander bezogen und für seine Zwecke ausgewertet hat, und das vielleicht in der folgenden Weise. Ausgangspunkt seiner Analyse scheint eine möglichst genaue Beschreibung der Phänomene gewesen zu sein, also nicht das, was er in der Tradition an Wissen vorfand. Dieses konnte Anlaß sein, eigene Beobachtungen anzustellen. Worauf es dann aber ankam, war die eigene Anschauung. In diesem Punkt erweist sich Ickelsamer als ein typischer Vertreter des Humanismus. Und so hat er versucht, die für die deutsche Sprache konstituierenden kleinsten Einheiten, kurz: die Laute, einmal durch die Abgrenzung voneinander zu identifizieren und dann durch eine möglichst exakte Bestimmung ihrer lautlichen Qualitäten zu beschreiben. Ein Beispiel, das auch den für seine Ausführungen charakteristischen Vortrag erkennen läßt: die Beschreibung des Lautes $/ \mathrm{n} /$ :

Das /n/ nennet Quintilianus ainen klingenden bůchstaben/

dann er treibet ainen athem durch die nasen/

das er dem nenner gleich im hirn klingt/

und rưrt die zung oben an den gůmen/

und wer ain schwach oder blöde hirn oder kopff hatt/

dem thůt dises bůchstabens nennung wehe (Müller 1882, 128). 
Die exakte Beschreibung der einzelnen Laute ist die Grundlage sowohl für die Erfassung der im Deutschen relevanten Laute als auch für ihre Klassifizierung.

Wie Ickelsamer bei der Erfassung der Laute vorgegangen ist, läßt der Vergleich mit einer Fibel erkennen, die etwa 50 Jahre zuvor auf den Markt gekommen ist - „um 1486“ (Kiepe). Hansjürgen Kiepe (1983) hat die Fibel gefunden und untersucht. Er kommt zu dem Ergebnis, daß in dieser Fibel zwar der Anspruch erhoben wird, die Laute des Deutschen zu erfassen, „von einer Lautlehre des Deutschen“ aber „eigentlich nicht die Rede sein kann“:

Das zeigt sich etwa an der Behandlung von $y$ und $z$. Die Schrift verweist darauf, $\mathrm{da} ß$,sy sind neulich gefunden“ (d. h. vor 1500 Jahren!) und „wir süllent sie nur schreiben (...), in den worten der kriechischen zungen und ebreischen“ (...), womit im Prinzip die Position der lateinischen Grammatik wiederholt wird. Gleichwohl wimmelt der deutsche Text natürlich von $y$ - und $z$-Schreibungen. Noch aufschlußreicher ist vielleicht, daß sch und $w$ gar nicht behandelt werden, obwohl der deutsche Text auch ohne sie nicht auskommen kann. Entsprechend bleibt das Problem des Umlauts, da ohne Vorbild im Lateinischen, gänzlich ungelöst (Kiepe 1983, 457).

Der Verfasser der Fibel hat also nur wiedergegeben, was er in der grammatischen Tradition vorfand, ohne sich zu vergewissern, ob das, was für das Lateinische galt, auch auf das Deutsche zutreffen konnte. Eine solche Gedankenlosigkeit ist Ickelsamer nicht unterlaufen. Er hat geprüft, ob das lateinische Alphabet zur Wiedergabe auch des deutschen Lautsystems geeignet ist und, wo dies nicht der Fall ist, es angepaßt und modifiziert. Das lateinische Alphabet blieb zwar die Basis, doch die Form, die es nun erhielt, war auf das Deutsche zugeschnitten (vgl. Ickelsamer in Müller 1882, 137-142).

Auf der Basis der Beschreibung und Erfassung der für das Deutsche relevanten Laute konnte Ickelsamer eine Klassifzierung vornehmen, die sich ausschließlich an den artikulatorischen Qualitäten der Laute orientierte. So unterschied er Vokale („die laut buchstaben“): fünf bzw. mit den Umlauten acht an der Zahl; die Konsonanten im eigentlichen Sinne des Wortes (die mitlautenden Buchstaben oder „mitstymmer") und „die gantz haimlichen oder stumm buchstaben": die Plosiven oder Verschlußlaute. Es handelt sich um die Laute, die „allain gar kain stymm“ (Müller 1882, 129) und darum schon von den Römern als „mutae“ (stumme) bezeichnet wurden.

Die Analyse des deutschen Lautsystems ist von der Nachwelt, aber auch von Ickelsamer selbst, ausschließlich als Grundlage seiner Lesemethodik angesehen worden. Das aber hieße, sie in ihrer Bedeutung unterschätzen. Denn in eben dem Maße, wie sie Grundlage der Lesemethodik war, ist sie auch Grundlage des Schreibunterrichtes, wie er in dem Gesamtkonzept bei Ickelsamer angelegt ist. Da aber aus dem Schreibunterricht die Ansätze eines umfassenderen Lehrplanes entwickelt werden (s. unten), kann mit Fug und Recht behauptet werden, daß die Lautanalyse für Ickelsamer die Grundlage des gesamten Unterrichts im Deutschen darstellt. 


\section{Der dem neuen Unterricht implizite Lehrplan}

Der Rahmen, den Ickelsamer seinem Gedankengebäude durch die Angabe sowohl der Bestimmung des Unterrichts als auch seiner Grundlegung gegeben hat, läßt darauf schließen, daß er sich auch Vorstellungen davon gemacht hat, wie das Ziel zu erreichen sei, in welchen Schritten dies zu geschehen habe und wie die Schritte geordnet werden können. Zwar hat er sich an keiner Stelle explizit dazu geäußert, doch reichen seine Bemerkungen aus, um so etwas wie einen zugrundeliegenden Lehrplan zu erschließen.

\subsection{Die lautanalytischen Übungen}

Ickelsamer läßt den Unterricht, wie er ihn sich vorstellt und vielleicht auch selber praktiziert hat, nicht gleich mit dem Lesen oder Schreiben beginnen. Er stellt an seinen Anfang so etwas wie ein Propädeutikum (zum folgenden Giesecke 1979, 55-57; 1992, 134-137; Rössing-Hager 1983, 543-547).

Ein solches Vorgehen ist grundsätzlich nicht neu. Denn auch der bisherige Unterricht hatte nicht gleich mit Übungen im Lesen oder Schreiben begonnen. Vielmehr hatten die Schüler, bevor sie überhaupt mit Buchstaben in Berührung kamen, erst einmal das Alphabet auswendig zu lernen (Göckelbecker 1933, $32 \mathrm{f}$.), und erst wenn sie dieses auswendig hersagen konnten - in der aus der lateinischen Tradition üblichen Reihenfolge und mit den Bezeichnungen, die für diese Tradition charakteristisch sind (A, be, ce, de, e usw.), konnte man daran denken, die dafür vorgesehenen Schriftzeichen einzuführen. Das Auswendiglernen des Alphabets war also ein eigenständiger Unterrichtsgegenstand, durchaus dem Lesen- und Schreibenlernen an die Seite zu stellen.

Ickelsamer hat ein solches Vorgehen abgelehnt mit der Begründung, daß es zu umständlich, darum zu langsam und mühsam sei und im übrigen nicht dazu geeignet, den eigenen Geist in Anspruch zu nehmen (Rössing-Hager 198, 543):

Das sy (die Kunst des Lesens) aber so wenig leüt lernen und können/ und sy ain yeder doch gern wissen und kónnen wolt/

wie man dann tåglich vil klagens hớrt/

von denen/ die solches in der jugent nit gelernt/

hie/ sag ich/ ists freilich kain andere vrsach/

dann das wenig Lerer/

die rechte kunst vnd weis/lesen zu leren/ kónnen und brauchen/

dann das gewơnliche Bůchstaben/

oder die nennung der Bůchstaben/ Be ce de etc.

ist den Alten vnd gewachßnen zů langksam und verdrüßlich/

wie dann solches auch kain kunst ist/

sonder nur ain gewonheit/ oder ain blinde erratung (Pohl 1979, A4v).

An die Stelle der geistlosen Memorierübungen stellte Ickelsamer die Übungen, die ihn bekannt gemacht haben: lautanalytische Operationen, die das Ziel haben, die Schüler mit den Lauten des Deutschen als der Grundlage für die Schriftzeichen bekannt zu machen. 
Begonnen wird bei den Wörtern der gesprochenen Rede, diese werden auf die während des Sprechens sich ereignenden lautlichen Veränderungen abgehorcht und immer dann, wenn sich eine lautliche Veränderung abzeichnet, wird das vorausgegangene Lautsegment abgetrennt, isoliert und mit anderen verglichen. Alle gleichlautenden Segmente ergeben jeweils einen Laut des Deutschen. In der Schrift von 1527, „Der rechten weis, aufs kürzist lesen zu lernen“, wird der Lernprozeß folgendermaßen beschrieben:

Aber also gehet es mit dem lesen vnnd den buchstaben zue/

das man ein wort ynn der rede absetzen mus yn seyne teyle (Pohl 1979, A3r).

Eigentlich wäre es nur konsequent gewesen, nicht erst bei den Wörtern, sondern schon bei gesprochenen Äußerungen zu beginnen, wie es ein moderner Phonologe machen würde. Doch Ickelsamer scheint ein Bewußtsein für Wörter bei Kindern vorausgesetzt zu haben, so daß ein solcher Analyseschritt sich erübrigte. In der „Teütschen Grammatica“ hat Ickelsamer das Verfahren an einem Beispiel verdeutlicht. Es geht um das Wort „Hans“:

da sein vier verenderung/ (...)

Zum ersten hơrt vnd vernymbt man ainen starcken athem/

wie man inn de hende haucht/ das ist das /h/

das haucht man auff den laut /a/

nach dem laut /a/ ainen klang durch die nasen/

vnnd zum letsten wirdt gehơrt ain junge tauben oder schlangen sibilen.

Ickelsamer resümiert:

Da ist kain tayl vnter disen vieren dem andern gleich/

und ist niemandts so vnverstendig vnd unmercksam/

der nit hören vnd mercken wolt/

was das sey das also den laut /a/ herauß athemet und haucht/

so er das wort selbs in seinen mundt nymbt/

vnnd merckt im nennen

wie vnd mitt welchem gerüst ain yede verenderung des worts gemacht wirdt

(Müller 1882, 132).

Ickelsamer hat also den Teil des Elementarunterrichts, der bisher dem Erlernen des Alphabets gegolten hatte, durch lautanalytische Übungen ersetzt und damit dem Elementarunterricht, soweit er das Sprachliche betrifft, insgesamt eine neue Grundlage gegeben.

\subsection{Die Übungen im Schreiben}

Es kam damals nicht selten vor, daß sich der Elementarunterricht auf Lesen und Rechnen beschränkte und Schreiben kein Thema war. Wenn ein Unterricht im Schreiben erteilt wurde, geschah dies in der Regel losgelöst vom Leseunterricht, oft auch in einem mehr oder weniger großen zeitlichen Abstand zu diesem und in vielen Fällen durch eine andere Lehrperson (Thomas 1985, 99 f.). Generell beschränkte er sich darauf, die Kinder anzuweisen, „gute teutsche Buchstaben zumachen", wie es in der Würtembergischen Kirchenordnung 
von 1559 treffend heißt (Vormbaum 1860, 160). Alles weitere blieb, wie gesagt, dem Lateinunterricht oder einem privaten Schreiblehrer überlassen. Man muß allerdings hinzufügen, daß zwei Aufgaben hinzukamen, die für uns heute nicht mehr selbstverständlich sind: einmal die schmuckvolle Ausgestaltung einzelner Buchstaben, die Kalligraphie, und dann auch noch die Beherrschung weiterer Schriften, außer der normalen Schreibschrift in der Regel die Fraktur, eine rein deutsche Spezialität, und eine Kanzleischrift (Ludwig in Vorbereitung (b)).

Ickelsamer muß sich von einem solchen Schreibunterricht weit entfernt haben. Aus der inneren Logik seines Ansatzes ergibt sich, daß auf die Erfassung, Beschreibung und Klassifizierung der für das Deutsche charakteristischen Laute die Einführung der ihnen entsprechenden Buchstaben erfolgen mußte (ausführlicher Vogel 1894, 48-50). Die Frage ist, ob diese lediglich durch Anschauung zur Kenntnis genommen wurden, so daß man dann gleich mit dem Lesen beginnen konnte, oder ob nicht doch die ersten Schreibübungen zwischengeschaltet wurden. Die damalige Unterrichtspraxis scheint sich mit der Anschauung begnügt zu haben. Denn nur so ist verständlich, daß man lesen lernen konnte, ohne vorher überhaupt einen Buchstaben geschrieben zu haben. Eine solche Möglichkeit ist bei Ickelsamer nicht grundsätzlich auszuschließen. Doch gibt es einige Indizien, die dafür sprechen, daß in seinen Vorstellungen das Schreiben dem Lesen voranzustellen ist. Einmal spricht er mehrfach an Stellen, an denen man es erwarten würde, tatsächlich von Schreibaktivitäten, und dann schiebt er zwischen die Laut- und die Leseübungen einige Bemerkungen zur Orthographie ein, also zum Schreiben, wenn auch nicht zum elementaren Schreiben. Dieser Abfolge entnehme ich, daß damit der Ort markiert ist, an dem die Schreibübungen zu plazieren wären.

Die Entscheidung, auf die lautanalytischen Übungen regelrechte Schreibübungen folgen zu lassen, wenn sie denn von Ickelsamer getroffen worden sein sollte, hätte gravierende Veränderungen der Unterrichtspraxis zur Folge gehabt. Es hätte sich die Frage, ob ein Schreibunterricht zu erteilen sei, überhaupt nicht mehr gestellt. Er wäre notwendig gewesen, weil der Leseunterricht ihn voraussetzte. Nicht nur die Abfolge von Schreib- und Leseunterricht wäre ein für alle Male geregelt gewesen, sondern beide wären auch in einen Zusammenhang miteinander gebracht und aufeinander bezogen worden. Schließlich wäre auch damit zu rechnen, daß sich der Schreibunterricht nicht mehr mit dem „Machen guter teutscher Buchstaben“ zufrieden gab, sondern sich öffnete, um weitere Aufgaben zu übernehmen, etwa das orthographisch und wohl auch das grammatisch richtige Schreiben (s. unten).

\section{3. Übungen im Lesen}

Wenn man in Rechnung stellt, daß im Lehrplan Ickelsamers lautanalytische Übungen (3.1.) und, wie wohl vorausgesetzt werden kann (3.2.), auch Übungen im elementaren Schreiben vorausgegangen sind, dann nehmen sich seine 
Ausführungen zum Unterricht im Lesen keineswegs so neu oder gar spektakulär aus, wie sie oft hingestellt werden. Sie halten sich durchaus im Rahmen des damals Üblichen.

In der grammatischen Tradition unterschied man den Lautwert der Buchstaben (lat. „potestas") sowohl von ihrer graphischen Form („forma“ oder "figura") als auch von ihrer Benennung („nomen“). Die Benennung war Gegenstand der Einführung in das Alphabet, die bei Ickelsamer durch lautanalytische Operationen ersetzt wurde. Mit der graphischen Form wurden die Kinder im Schreibunterricht vertraut gemacht. Die Kinder anzuleiten, den Buchstaben die ihnen entsprechenden Lautwerte zuordnen zu können, blieb dem Leseunterricht vorbehalten (zum folgenden Vogel 1894, 52-57).

Lesen war für Ickelsamer wie für seine Zeitgenossen: den Buchstaben eine Stimme geben, wie er mehrfach diesen Vorgang charakterisierte, und Lesenlernen war der Erwerb der Fähigkeit, die Buchstaben zum Erklingen zu bringen. Man hat, wie die ganze Antike hindurch und auch noch im Mittelalter (Balogh 1927; Saenger 1982), nicht still vor sich hin, sondern laut gelesen, d. h. mit dem Mund (oral) und für das Ohr bestimmt (aural), und das - nach allem, was wir inzwischen wissen - nicht etwa, weil man es nicht besser konnte, sondern weil erst das über das Ohr Gehörte Grundlage für das Verständnis des Gelesenen war. Den Sinn einer Aussage konnte man nur dem gesprochenen und infolgedessen gehörten Wort entnehmen. Der Prozeß des Verstehens war also aural, d. h. an das Ohr gebunden (Ludwig in Vorbereitung (a)).

Ickelsamer unterschied sich von seinen Zeitgenossen nicht im Ziel, sondern in der Methode des Leseunterrichtes. Bis dahin hatte man Lesen nach der Buchstabiermethode gelernt (Göckelbecker 1933, 27-40). Jedem Buchstaben wurde eine bestimmte Silbe zugeordnet, deren erster Laut genau dem Laut entsprach, den der Buchstabe repräsentieren sollte. So entsprach dem Buchstaben „b" die Silbe /be/, dem „c“ die Silbe /ce/ und so fort. Die jeweiligen Benennungen der Buchstaben waren im Alphabet festgeschrieben. Nach einem solchen Prinzip verfährt auch heute noch die Telephonauskunft, wenn der Name einer Person, dessen Schreibung unklar ist, bestimmt werden soll. Nur hat man sich auf andere Benennungen geeinigt: Eigennamen stehen an Stelle von Silben.

Ickelsamer nimmt von der Buchstabiermethode Abstand und ersetzt sie durch die Lautiermethode, wie man seine Methode später bezeichnet hat (Göckelbecker 1933, 40-67). Das Verfahren ist einfach und ergibt sich aus der inneren Logik seines Lehrplanes. Nachdem die Schüler und Schülerinnen bis zu dem Zeitpunkt, an dem der Leseunterricht einsetzte, in den lautanalytischen Übungen mit den im Deutschen vorkommenden Lauten und vermutlich im Schreibunterricht mit den dem Deutschen eigenen Buchstaben vertraut gemacht worden waren, blieb es dem Leseunterricht vorbehalten, sie anzuleiten, die Laute auf die Buchstaben zu beziehen. Von der Wiedergewinnung einzelner Laute bis zum Erlesen ganzer Wörter war nur noch ein kleiner Schritt. Ickelsamer hat ihn so beschrieben: 
So bald nun ainer der namen vnd figur der Bůchstaben auff dise weis berichtet ist/ der fach an zůlesen/ (...)

Er lese (...) vnd nenne die bůchstaben also/

das er in ainem wort nit abbrechen biß ers gar hinauß liset/

vnd laß kainen bůchstaben faren/

biß er den andern ergriffen hat/

vnd henck es alles anainander/ wie die ring an ainer ketten (Müller 1882, 137).

Vogel hat den Unterschied zur alten Methode des Lesenlernens treffend so beschrieben: „I(ckelsamer) gebührt das Verdienst, das nomen durch die potestas, den Laut, ersetzt zu haben“ (1890, 40). „An die Stelle der Buchstabennennung muss die Lautnennung treten" (ebd. 39).

Die Abfolge von Lautanalyse, Schreiben und Lesen war irreversibel und läßt deutlich eine Richtung erkennen, die mit dem Lesenlernen keineswegs abgeschlossen ist. Sie öfnet sich weiteren unterrichtlichen Aufgaben und damit für ein ganzes Curriculum. Mit den neuen Aufgaben wird aus dem Schreib- und Leseunterricht ein Deutschunterricht, aus den Schreibund Rechenschulen des späten Mittelalters erst eigentlich eine deutsche Schule.

\subsection{Orthographische Übungen}

Der Schritt vom Schreib- und Leseunterricht zu einem Unterricht der deutschen Sprache, der entscheidende Schritt zum Deutschunterricht, erfolgte nicht und konnte auch nicht über den Leseunterricht erfolgen. Wenn Lesen in damaliger Zeit nicht mehr war als die Lautierung der Buchstaben, weil den geschriebenen Wörtern sozusagen eine Stimme verliehen werden mußte, dann bestand der Leseunterricht im wesentlichen aus dem Erwerb der Fähigkeit, solche Lautierungen von Wörtern vornehmen zu können. Eine solche Fähigkeit konnte zwar geübt werden. Darum hat Ickelsamer seinen Schriften einige Übungstexte angefügt. Doch ausbaufähig war ein solcher Leseunterricht nicht.

Ausbaufähig war dagegen der Schreibunterricht. Wenn es das Ziel des Unterrichtes überhaupt war, die Kinder anzuleiten, ,recht und gut teütsch zu reden/und schreiben“, dann konnte man sich nicht mit dem „Machen guter deutscher Buchstaben“ zufrieden geben. Um „Deutsch richtig zu schreiben“ bedurfte es an erster Stelle der richtigen Schreibung der Wörter. Es ist kaum anzunehmen, daß die Rechtschreibung vor Ickelsamer schon Bestandteil des Schreibunterrichtes war. Im Gegenteil. Einmal war der Schreibunterricht noch nicht mit rhetorischen Übungen verbunden, in denen die Produktion von Texten gelernt werden konnte, die die Notwendigkeit, die Wörter richtig zu schreiben, hätte vor Augen führen können. Dann ist zu berücksichtigen, daß eine einheitliche Schreibung der Wörter weder in Sicht, noch zu erwarten war. Ickelsamer beklagt dies ausdrücklich: 
(...) das unrecht schreiben der wörter und buchstaben/

ist in diser sprach (der deutschen Sprache OL.) so gemain/

das der gemain brauch nun muß kunst sein/

darzů so ists den Teütschen nit wol müglich recht zuschreiben/

dieweil sy/ wie gsagt/ nichts wenigers verstehn/

dann jre teütsche sprach (Pohl 1979, C6v).

So ist auch nur konsequent, daß Ickelsamer die Rechtschreibung als eine wichtige Aufgabe des Elementarunterrichts begriffen hat. Was für den Lateinunterricht selbstverständlich war, konnte für den Unterricht des Deutschen nicht falsch sein:

Bey den Lateinischen wirdt die Orthographia/

das ist/ recht (!) bůchståbisch schreiben/

so eben vnd fleyssig gehalten/

das ainer der gantzen lateinischen kunst vnwissend würdt geachtet/

der nur ainen bůchstaben vnrecht/

oder ainen zůuil oder zů wenig setzet/

warumb soll es dann bey den Teütschen gleich gelten/

man schreib recht oder falsch?

kündt man doch dise sprach so wol regulieren als die Hebraisch/

Ghriechisch oder Lateinisch sein.

Und so fordert er „ain yeden“ dazu auf:

das er vmb rettung willen unser gemainen Teütschen sprach/ (...)

gantz aigentlich wôl auffmercken/

wa er ainen yeden Bůchstaben am rechtisten

vnd subtilisten setzen und gebrauchen soll/

vnd nitt also vnbesunnen ainen yeden überal gebrauchen (Müller 1882, $130 \mathrm{f}$.).

Stand die Notwendigkeit orthographischer Übungen für Ickelsamer außer Frage, so stellte sich die Frage nach ihrer Durchführung. Auch dazu hat er sich geäußert, wenn man seine eher systematischen Anmerkungen als methodische Ratschläge verstehen darf (so schon Giesecke 1992). Ickelsamer unterschied zwei Zustände der deutschen Rechtschreibung: einen idealen, der von klaren Regelungen bestimmt ist, und einen anderen des „gemainen brauchs“, in dem viele Ungereimtheiten zu verzeichnen sind, der ihm aber reformierbar zu sein schien. In Entsprechung zu den beiden Zuständen wird der Unterricht in zwei Schritten vollzogen. In einem ersten Schritt sollten die Schüler und Schülerinnen mit den beiden Grundregeln der deutschen Rechtschreibung bekannt gemacht werden. Man sollte annehmen, daß Ickelsamer - getreu seinem theoretischen Ansatz - von den Laut-Buchstaben-Korrespondenzen ausgegangen wäre. Das ist aber nicht geschehen. Ickelsamer fängt bei den lexikalischen Bedeutungen an. Als erstes solle man auf die „Bedeutung“ („Signification“) und den Aufbau („Composition“) der Wörter achten. Denn aus der Bedeutung eines Wortes oder seiner Teile ließen sich $u$. U. schon wichtige Hinweise für seine Schreibung gewinnen. Daß dem lexikalisch-semantischen Prinzip der Vorrang vor dem phonetischen eingeräumt wird, ist verständlich, wenn man bedenkt, da $\beta$ es damals keine einheitliche Hochsprache gab, so daß die aus- 
schließliche Anwendung des phonetischen Prinzips zu den unterschiedlichsten Schreibungen Anlaß gegeben hätte. Das wird an einem Beispiel verdeutlicht:
Das wort harbant/
reden etlich das /b/ so waich das es lauttet harwant/
ja etlich nennens harwet/
Wer nu nit waist die signification und bedeütung dises worts/
Nămlich/ das gesagt ist von ainem band da man das har mit bindet/
wie kan ers recht schreiben oder reden? (Müller 1882, 132).

Erst nachdem man sich der Bedeutung eines Wortes vergewissert hatte, sollte die Lautung zum Zuge kommen:

\author{
Die ander (Regel OL.)/ \\ Das er das selbig wort oder seine tayl/ das ist/ \\ die bůchstaben vor in seine oren nemel \\ und frag seine zungen wie es kling/ \\ hart oder waich/ \\ vnd was es aigentlich für laute hab (ebd.).
}

Die Vertrautheit mit den beiden Grundregeln der deutschen Orthographie scheint Ickelsamer sehr am Herzen gelegen zu haben. Daraus kann man auf das Vertrauen schließen, das er dem Regelwissen für den Unterricht - auch in der Rechtschreibung - entgegenbrachte.

Waren die Kinder in der Lage, die beiden Grundregeln anzuwenden, dann konnten sie sich zwar eine Vorstellung von einem idealen Zustand der deutschen Rechtschreibung machen, doch reichte diese nicht aus, dem realen $\mathrm{Zu}$ stand gerecht zu werden. Dazu bedurfte es der Kenntnis weiterer Regeln, von denen Ickelsamer nur die Verdoppelung von Buchstaben diskutiert hat.

\title{
3.5. Übungen in der deutschen Grammatik
}

Wenn es Aufgabe des Unterrichts der deutschen Sprache sein sollte, die Kinder anzuleiten, „recht und gut teütsch zu reden/ und schreiben“, dann konnte man auch nicht bei der richtigen Schreibung der Wörter stehen bleiben, dann mußte der Schritt von der Orthographie zum richtigen und guten Gebrauch der Wörter in der Rede getan werden, also der Schritt hin zur Grammatik. Insofern war es nicht mehr als folgerichtig, wenn Ickelsamer sich für einen Unterricht einsetzte, in dem auch die Grammatik des Deutschen ein Gegenstand war (ausführlich Rössing-Hager 1983 und vor allem Vogel 1894, 58-76).

Grammatische Übungen gab es bisher nur im Lateinunterricht der weiterführenden Schulen, der Pfarr-, Stadt- oder Lateinschulen. Ihr Gegenstand war nicht eine deutsche, sondern ausschließlich die lateinische Grammatik. Im Elementarunterricht war Grammatik kein Thema, es gab noch nicht einmal eine deutsche Grammatik. Ein solcher Zustand war für Ickelsamer nicht hinnehmbar, und so forderte er nicht nur, daß, was für den Lateinunterricht selbstverständlich war, auch für einen Unterricht der deutschen Sprache zu gelten habe, sondern machte sich auch selber ans Werk und schrieb ein ganzes Büchlein zu 
diesem Thema, eben seine „Teutsche Grammatica“. Ausdrücklich merkt er an, daß der grammatische Teil für ihn Anlaß war, das Buch überhaupt zu schreiben:

Disen tail der Grammatic/

so in diesem meinen bưchlin gehandelt/

hab ich den besten vnd nützisten sein gedacht/

vnd deßhalben meinen geringen dienst gern dazů thon (Müller 1882, 122).

Ickelsamers Grammatik ist „ain teutsche Grammatica“, eine Grammatik der deutschen und nur der deutschen Sprache. Das unterscheidet seine Grammatik von allen Grammatiken vor ihm. Er hat wie kein anderer klar erkannt, daß es nicht angehen kann, den Donat oder den Priscian lediglich ins Deutsche zu übersetzen (Grubmüller 1983; Puff 1996):

Welcher aber ain lateinische Grammatica schlecht (schlicht=einfach) teütschen wil/ was sy im latein gibt des Grammatica würdt den teütschen

seltzamer vnd vnbekandter sein/

dann ain Lateinische/ oder villeicht ain Chalecutische (Müller 1882, $121 \mathrm{f}$.).

Eine Grammatik, die dem Anspruch genügen sollte, eine deutsche zu sein, mußte auf die Eigentümlichkeiten des Deutschen eingehen und also von Grund auf neu geschrieben werden. Ickelsamer hat dazu den Anfang gemacht.

Ickelsamers Grammatik ist darüber hinaus eine pädagogische Grammatik. Sie ist nicht nur eine „kunst“, sondern zugleich auch eine „unterweysung“, „recht und gut teütsch zu reden/ und schreiben“. Darum erhebt sie auch nicht den Anspruch, eine vollständige Beschreibung der grammatischen Verhältnisse im Deutschen zu sein, sondern kann unter den Stücken einer deutschen Grammatik diejenigen auswählen, die für ihre Zwecke als notwendig erachtet werden, aber auch - das ergibt sich aus ihrem Anspruch - andere unbeachtet lassen. Einer solchen Beschränkung fällt die ganze Formenlehre (Morphologie) des Deutschen zum Opfer. Ickelsamer ist der Auffassung, daß die Bildung der Wortformen zuhause gelernt worden ist und darum in der Schule nicht mehr gelernt zu werden braucht:

dann der schaft mit vil arbait wenig nutz/

der die teütschen leren will/

wie sy sagen vnd reden sollen/

der Hans/ des Hansen etc.

Ich schreib ich habe geschriben etc.

Das lernen die kinder besser von der můter/

Dann auß der Grammatic (Müller 1882, 120).

Als Gegenstände grammatischer Übungen bleiben demnach übrig: die acht Redeteile und die Syntax, und diese sind es auch, aus denen sich nach den Vorstellungen Ickelsamers eine deutsche Grammatik zusammensetzt:

Der aber die acht tayl der rede recht verteütschet vnd erklärt mit jren accidentijs vnd zůgeho̊rungen zum rechten gründtlichen verstandt der Teütschen wörtern vnd redel 
sampt ainer gůten teütschen Syntaxi und Construction/

das ist/

gantzer versamelter vnd rechter kunstmåssiger teütscher rede/

das wer auch billich ain teütsche Grammatica zů nennen (Müller 1882, 120).

\section{6. Übungen in der Bestimmung der Herkunft und Zusammensetzung deutscher Wörter (Etymologie und Wortbildung)}

Ickelsamer hatte dem Unterricht der deutschen Sprache zwei Ziele gesetzt: die Kinder sprachlich so weit zu fördern, daß sie erstens „recht und gut teütsch zureden/ und schreiben" und zweitens daß sie ,jre sprach bas (besser OL.) verstehn lernen". Es ging also sowohl um die Beherrschung der deutschen Sprache in Wort und Schrift als auch um ,ainen künstlichen verstand der gantzen teütschen wörter sprach art und weiß“, d. h. um Sprachverständnis. Dem ersten Lernziel dienten die orthographischen und grammatischen Übungen sowie der Elementarunterricht im Lesen und Schreiben, dem zweiten die Betrachtung der Wörter, vornehmlich unter dem Gesichtspunkt ihrer Herkunft (Etymologie) und Bildung (Komposition):

Es ist in allen sprachen/

glaub ich/

kaum ain lieblicher ding/

dann die Etymologias vnd Composition der wörter erkennen und verstehn/

dann es ist so künstlich ding/

das gleich etliche tieffe gehaimnuß allain vnter den Bůchstaben verborgen ligen

(Müller 1882, 147).

Herkunft und Bildung der Wörter sind für Ickelsamer also nur deshalb von Interesse, weil sie ihm Aufschluß über die Bedeutung der Wörter zu geben scheinen. Im Grunde geht es ihm um eine Wortsemantik.

Theodor Vogel nimmt an, daß Ickelsamer, ,an die Stelle der Lehre von den Wortformen die Lehre von der Wortbildung und Wortbedeutung setzt" $(1894,62)$. Das ist zwar nicht mehr als eine Vermutung. Denn Ickelsamer hat sich an keiner Stelle in diesem Sinne geäußert. Doch hat die Vermutung etwas für sich. Träfe sie zu, dann könnten wir feststellen, daß die Flexionsmorphologie nicht einfach weggefallen ist, sondern durch einen Gegenstand ersetzt wird, der bisher noch nie im Unterricht - zumindest im Unterricht an Elementarschulen - Gegenstand war.

Mit der Aufnahme etymologischer, kompositorischer und semantischer Fragen hat Ickelsamer den Rahmen des Grammatikunterrichtes gesprengt. Unterricht der deutschen Sprache war nicht wie bisher nur Unterricht im Lesen und Schreiben, aber auch nicht nur Grammatikunterricht, wie man Ickelsamer leicht unterstellen kann, sondern ging in letzter Konsequenz weit darüber hinaus. Er wurde in den Vorstellungen Ickelsamers zu dem, was er eigentlich bezeichnete: zum Unterricht der deutschen Sprache schlechthin: 
Ja das in summa kain so gering wort/

in der ganzten sprach sey/

das nit seinen namen/ von seinem ampt/

auß aigner sonderlicher gehaymnuß und bedeütung hab/

Vnnd das auch kain sprach/

die teütsch sonderlich/

gantz lauter/

sonder sein all vnter ainander vermischet/

Darumb auch kaine one die andern

volkomlich verstanden móg werden.

Vmb solcher grosser nutzbarkait/

vnnd feiner lieblichait willen/

solten ye die teütschen jre sprach bas verstehn lernen/

vnd solt kain wort sein

des vrsprung und bedeütung sy nit wisten (Müller 1882, 148 f.).

\section{Ergebnisse der Untersuchung}

Blickt man zurück auf den Verlauf des Unterrichts, wie ihn sich Ickelsamer mag vorgestellt haben, so läßt sich feststellen, daß ein reich gegliedertes Curriculum vorliegt. Es reicht von den elementaren Übungen in der Bestimmung der Laute des Deutschen, der Einführung in das Schreiben und Lesen über orthographische und grammatische Übungen bis hin zur Erörterung der Bedeutung und Etymologie von Wörtern. Vergleicht man jedoch, was Ickelsamer ausgeführt hat, mit dem Ziel und der Aufgabe, die er dem Unterricht der deutschen Sprache gesetzt hatte, dann wird man feststellen, daß die Durchführung hinter dem Ziel zurückbleibt und das Curriculum unvollständig bleibt.

Nicht eingearbeitet ist das „gute Schreiben“, von dem neben dem „rechten Schreiben" die Rede war. Stilübungen, in denen neben dem orthographischen und dem grammatisch korrekten Schreiben auch das der Situation angemessene, d. h. dem dargestellten Sachverhalt, dem zu erwartenden Leser und der eigenen Intention angepaßte Schreiben gelernt und geübt werden konnte, scheinen in den Vorstellungen Ickelsamers keine Rolle gespielt zu haben. Sie werden erst im 19. Jahrhundert den Schreib- und Aufsatzunterricht bestimmen (Ludwig 1988; 1998; Abraham 1996). Ickelsamers Vorstellungen sind mehr an dem richtigen Deutsch orientiert. Die Grammatik hat Vorrang vor der Stilistik, auch wenn stilistische Aspekte im Gewand rhetorischer Fragestellungen immer wieder berührt werden.

Nicht eingearbeitet ist schließlich auch das „recht und gut teütsch reden“, der ganze Bereich des mündlichen Gebrauchs der deutschen Sprache oder, wie es im Jargon der Deutschlehrer heißt, der mündliche Ausdruck. Allerdings kann man ihn bei einigem guten Willen extrapolieren. Die Lautanalysen am Anfang des Curriculums bewegen sich ausschließlich im mündlichen Bereich, dem Gesprochenen und Gehörten. Die Ausführungen zur Grammatik des Deutschen und zu den Bedeutungen deutscher Wörter sind keineswegs für das Schriftliche spezifisch und können ohne weiteres auf den mündlichen Ge- 
brauch des Deutschen angewendet werden. Aber selbst dann wäre ein deutliches Ungleichgewicht zwischen dem Schreiben und Reden festzustellen.

Die Tatsache, daß man feststellen kann, daß die Durchführung hinter dem Ziel zurückbleibt, schmälert aber in keiner Weise das Verdienst Ickelsamers. Es bleibt ihm die Anerkennung dafür, als erster eine Idee von einem Unterricht der deutschen Sprache entwickelt, der Öffentlichkeit vorgestellt und damit den Deutschunterricht überhaupt erst einmal auf den Weg gebracht zu haben.

Neu ist das Ziel, das Ickelsamer dem Unterricht an den Elementarschulen gesetzt hat und das weit über das hinaus geht, was damals im Elementarunterricht erreicht werden sollte. Es kam nicht mehr nur auf das Schreiben deutscher Wörter und Sätze und auch nicht mehr nur auf das laute Lesen deutscher Texte an, sondern auf den richtigen und angemessenen Gebrauch und eine gründliche Kenntnis der deutschen Sprache. Die Beschränkung auf das Schriftliche wird aufgehoben. Die Beherrschung des Deutschen schlechthin wird angestrebt, und das schließt grundsätzlich das Mündliche mit ein.

Neu ist auch die Öffnung des Lehrplanes für alle Gegenstände, die mit Sprache zu tun haben. Mit einer solchen Öffnung wird der Lehrplan erst eigentlich begründet. Die Öffnung geht, wie ausgeführt, nicht vom Lese-, sondern vom Schreibunterricht aus. Er ist es, der ein Curriculum des Deutschen konstituiert.

Neu ist schließlich und vor allem auch die Grundlegung des Unterrichts. Wenn man die Lautanalyse, wie das auch Ickelsamer getan hat, nur mit dem Leseunterricht in Verbindung bringt, ist das nur die halbe Wahrheit. So wie sie den Leseunterricht begründet, so begründet sie auch und in demselben Maße den Schreibunterricht. Im Grunde begründet sie das gesamte Curriculum. Löst man sie aus der ausschließlichen Bindung an den Leseunterricht, dann wird die Rolle erst eigentlich erkennbar, die sie im Curriculum Ickelsamers spielt. Sie ist ihr Fundament.

Was Ickelsamer über seine Zeit hinaus auszeichnet, ist die Tatsache, daß er als erster einen Entwurf des Deutschunterrichts vorgelegt hat, der zu seiner Zeit nur programmatischen Charakter hatte, verglichen aber mit dem, was aus dem Deutschunterricht im Verlauf mehrerer Jahrhunderte geworden ist, erstaunlich realistische Züge aufweist. Der Deutschunterricht ist - nach den hier vorgenommenen Rekonstruktionen - in seinen Grundzügen von Valentin Ickelsamer entworfen worden.

\section{Literatur}

Abraham, Ulf (1996): StilGestalten. Geschichte und Systematik der Rede vom Stil in der Deutschdidaktik (RGL 161), Tübingen: Niemeyer.

Balogh, J. (1927): „Voces Paginarum“. Beiträge zur Geschichte des Lesens. In: Philologus $82,84-109$ und $202-240$. 
Giesecke, Michael (1980): Schriftspracherwerb und Erstlesedidaktik in der Zeit des „gemein teutsch" - eine sprachhistorische Interpretation der Lehrbücher Valentin Ickelsamers. In: OBST 11 (Schriftspracherwerb), 40-58.

- (1992): Alphabetisierung als Kulturrevolution. Leben und Werk V. Ickelsamers (ca. 1500-ca. 1547). In: Sinnenwandel, Sprachwandel, Kulturwandel. Studien zur Vorgeschichte der Informationsgesellschaft (stw 997), Frankfurt a. M.: Suhrkamp, S. $122-185$.

Göbelbecker, L. F. (1937): Entwicklungsgeschichte des ersten Leseunterrichts von 1477-1532, Kempten u. Leipzig.

Grubmüller, Klaus (1983): Der Lehrgang des Triviums und die Rolle der Volkssprache im späten Mittelalter. In: Möller, B./Patze, H./Stackmann, K. (Hgg.), S. 371-397.

Jellinek, Max Hermann (1913f.): Geschichte der deutschen Grammatik von den Anfängen bis auf Adelung, 2 Bde, Heidelberg.

Kiepe, H. (1983) Die älteste deutsche Fibel. Leseunterricht und deutsche Grammatik um 1486. In: Moeller/Patze/Stackmann (Hgg.), S. 435-461.

Kintzinger, Martin (1990): Das Bildungswesen in der Stadt Braunschweig im hohen und späten Mittelalter, Köln: Böhlau.

Ludwig, Otto (1988): Der Schulaufsatz. Seine Geschichte in Deutschland, Berlin/New York: de Gruyter.

- (1998); Alphabetisierung und Volksschulunterricht im 19. Jahrhundert. Der Beitrag der Schreib- und Stilübungen. In: Cherubim, C./Grosse, S./Mattheier, K. (Hgg.). Sprache und bürgerliche Nation, Berlin/New York: de Gruyter, S. 148-166.

- (in Vorbereitung (a)): Lesen und Schreiben in der Antike.

- (in Vorbereitung (b)): Drei Mal Schreiben: Schriftlichkeitsstandards im Schreibunterricht des ausgehenden Mittelalter bis zur Gegenwart.

Moeller, Bernd/Patze, Hans/Stackmann, Karl (Hgg.) (1983): Studien zum städtischen Bildungswesen des späten Mittelalters und der frühen Neuzeit, Göttingen (Abh. d. Akad. d. Wiss. in Göttingen, Phil.-H. 3, 137).

Müller, Johannes (1882): Quellenschriften und Geschichte des deutschsprachigen Unterrichts bis zur Mitte des 16. Jahrhunderts, Gotha (Nachdruck Hildesheim: Olms 1969).

Noll, Heinrich (1935): Der Typus des religiösen Grammatikers im 16. Jahrhundert Dargestellt an V. Ickelsamer (Diss.), Marburg.

Pohl, Karl (1971): Valentin Ickelsamer, Stuttgart: Klett.

Puff, Helmut (1995): „Von dem schlüssel aller künsten nemblich der Grammatica“. Deutsch im lateinischen Unterricht, Tübingen.

Rössing-Hager, Monika (1983): Konzeption und Ausführung der ersten deutschen Grammatik. Valentin Ickelsamers „Ein Teütsche Grammatica“. In: Literatur und Laienbildung im Spätmittelalter und in der Reformatonszeit. Hg. von Grenzmann, Ludger/Stackmann, Karl. Stuttgart, S. 534-556.

Saenger, Paul (1982): Silent Reading. Its Impact on Late Medieval Script and Society. In: Viator 13, 367-414.

Socin, Adolf (1888): Schriftsprache und Dialekte im Deutschen nach Zeugnissen alter und neuer Zeit. Heilbronn (Nachdruck Hildesheim: Olms 1970).

Thomas, Rosalind (1989): Oral Tradition and Written Record in Classical Athens. Cambridge; Univ. Press.

Vogel, Theodor M. (1894): Leben und Verdienste Valentin Ickelsamers. Ein Beitrag zur Geschichte der speziellen Methodik (Diss.), Leipzig.

Vormbaum, Reinhold (Hg.) (1860): Die evangelischen Schulordnungen des sechszehnten Jahrhunderts. In: Evangelische Schulordnungen Bd. 1, Gütersloh: Bertelsmann.

Adresse des Verfassers:

Prof. Dr. Otto Ludwig, Universität Hannover, Seminar für deutsche Literatur und Sprache, Königsworther Platz 1b, 30167 Hannover 